\title{
The X-ray spectral evolution of Cygnus X-2 in the framework of bulk Comptonization
}

\author{
R. Farinelli ${ }^{1}$, A. Paizis ${ }^{2}$, R. Landi ${ }^{3}$, and L. Titarchuk ${ }^{1,4}$ \\ ${ }^{1}$ Dipartimento di Fisica, Università di Ferrara, via Saragat 1, 44100 Ferrara, Italy \\ e-mail: farinelli@fe.infn.it \\ 2 INAF - IASF, Sezione di Milano, via Bassini 15, 20133 Milano, Italy \\ 3 INAF - IASF, Sezione di Bologna, via Gobetti 1, 40100 Bologna, Italy \\ 4 NASA - GSFC, Greenbelt, MD 20771, USA
}

Received 19 June 2008 / Accepted 16 January 2009

ABSTRACT

\begin{abstract}
Context. Strong theoretical and observational support exists that the spectral evolution of neutron-star LMXBs, including transient hard X-ray tails, can be explained by the interplay between thermal and bulk motion Comptonization. The introduction of a new XSPEC Comptonization model, COMPTB, including thermal and bulk Comptonization, has provided additional support to this interpretation.

Aims. We used CомPтв to investigate the spectral evolution of the neutron-star LMXB Cyg X-2 along its Z track. We selected a single source to trace in a quantitative way the evolution of the physical parameters of the model.

Methods. We analyzed archival broad-band BeppoSAX spectra of Cyg X-2. Five broad-band spectra were newly extracted by using information about the source position in the $\mathrm{Z}$ track described in the colour-colour and colour-intensity diagrams.

Results. We fitted the spectra of the source with two COMPTB components. The first one, with a bulk parameter $\delta=0$, represents the dominant component of the overall source broad-band spectrum and its origin is related to thermal upscattering (Comptonization) of cold seed photons by warm electrons in a high opacity enviroment. We attribute the origin of these seed photons to the section of the disk that illuminates the outer coronal region (transition layer) located between the accretion disk itself and the neutron-star surface. The physical properties of this thermal component are roughly constant with both time and inferred mass accretion rate. The second COMPTB model describes the overall Comptonization (thermal plus bulk, $\delta>0$ ) of hotter seed photons that originate in both the inner transition layer and at the neutron-star surface. This component is more significant in the horizontal branch of the colour-colour or hardness-intensity diagram and progressively disappears towards the normal branch, where a pure blackbody spectrum is observed. Conclusions. The spectral evolution of Cyg X-2 is studied and interpreted in terms of changes in the innermost environmental conditions of the system, leading to a variable thermal-bulk Comptonization efficiency.
\end{abstract}

Key words. stars: individual: Cygnus X-2 - stars: neutron - X-rays: binaries - accretion, accretion disks

\section{Introduction}

The bright persistent Low Mass X-ray Binary (LMXB) Cyg X-2 is inferred to be a Galactic source that hosts a neutron star (NS) belonging to the $\mathrm{Z}$ class, because of the $\mathrm{Z}$ shape it displays in its colour-colour (CD) and hardness-intensity diagram (HID). The different parts of the diagram are referred to as the horizontal branch (HB) at the top of the Z, the normal branch (NB) along its diagonal, and the flaring branch (FB) at the base of the $\mathrm{Z}$ diagram. In reality, $\mathrm{Z}$ sources move continuously within this diagram, and the source position within the $\mathrm{Z}$ is probably related to the mass accretion rate which increases from the HB to the FB (Hasinger et al. 1990).

The X-ray spectrum of Cyg X-2, and of persistent bright NS LMXBs in general, has usually been described below $20 \mathrm{keV}$ by the sum of a soft and a hard component. The soft component is interpreted as emission from the accretion disc (Eastern model, Mitsuda et al. 1984, 1989) or as originating close to the NS (Western model, White et al. 1986, 1988), whereas the hard component is probably produced by Comptonization of NS and/or disc emission by a hot plasma (so-called "corona") electrons. These two models describe equally well the spectra of NS LMXBs below about $20 \mathrm{keV}$. The first studies of the Cyg X-2 X-ray spectrum above $20 \mathrm{keV}$ were performed by balloon detectors (Peterson 1973). Interestingly, an unexpected hardening was observed in the spectrum of the source, which was fitted by a powerlaw (PL) with photon index of 2.8 (Maurer et al. 1982) or 1.9 (Ling et al. 1996). It soon became clear that simultaneous broad-band observations of the X-ray spectrum were needed to investigate the nature of this spectral flattening.

The advent of broad-band X-ray missions, such as BeppoSAX, RXTE, and INTEGRAL, revealed that many similar spectral hardenings (so-called "hard tails") occur in Z sources: Cyg X-2 (Frontera et al. 1998; Di Salvo et al. 2002, hereafter DS02), GX 17+2 (Di Salvo et al. 2000; Farinelli et al. 2005), GX 349+2 (Di Salvo et al. 2001), Sco X-1 (D'Amico et al. 2001; Di Salvo et al. 2006), GX 5-1 (Asai et al. 1994; Paizis et al. 2005), GX 340+0 (Lavagetto et al. 2004). A hard tail has also been discovered in the bright atoll source GX 13+1 (Paizis et al. 2006, hereafter P06).

The spectra of these sources and in particular the hard $\mathrm{X}$-ray tails have been studied extensively by focusing on single sources, and on interpretation with phenomenological models (see Barret 2001; Di Salvo \& Stella 2002, for a review on NS LMXB spectra). To investigate these sources in a unified physical scenario for the less well studied energies above $20 \mathrm{keV}$, 

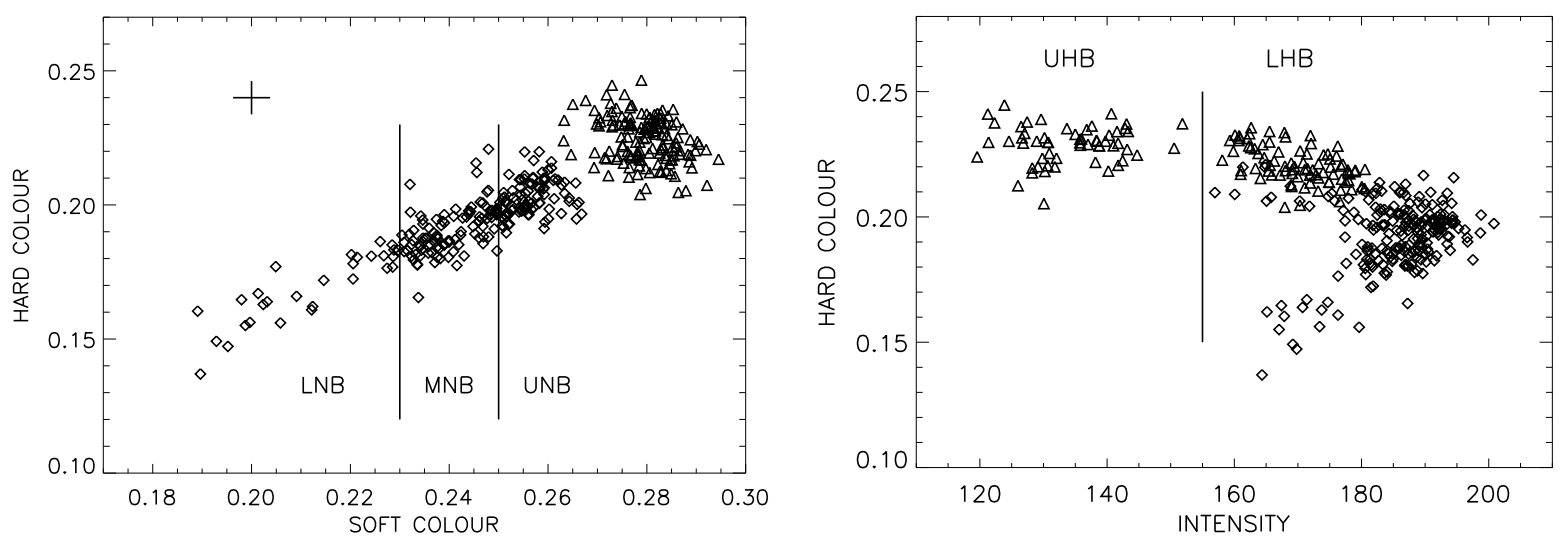

Fig. 1. Colour-colour diagram (left panel) and hardness-intensity diagram (right panel) of Cyg X-2 during the 1996 (triangles) and 1997 (diamonds) observation. The hard colour is defined as the $7-10.5 \mathrm{keV} / 4.5-7 \mathrm{keV}$ count-rate ratio, while the soft colour is defined as the 4.5-7 keV/1.4-4.5 keV count-rate ratio. For the hardness-intensity diagram, the intensity is given by the 1.4-10.5 keV count rate. A typical error bar is shown in the left panel. The regions of the branches of the $\mathrm{Z}$ pattern for which spectral analysis was carried out are also shown.

P06 studied the long-term, average hard X-ray ( $>20 \mathrm{keV})$ spectra of twelve bright NS LMXBs (six Z and six atoll sources), using data from IBIS instrument on board INTEGRAL (Winkler et al. 2003). Merging their results with those of Falanga et al. (2006) for the atoll source 4U 1728-34 (GX 354-0), P06 identified four main spectral states for NS LMXBs (see Fig. 4 in P06): a low/hard state (GX 354-0), a hard/PL state (H 1750-440 and $\mathrm{H}$ 1608-55), an intermediate state (where the hard X-ray tail appears, e.g., Cyg X-2, Sco X-1, GX 5-1, GX 17+2), and a soft state (e.g., GX 3+1, GX 9+1, GX 9+9). The different spectral states, including the hard tails, could be reproduced as the result of interplay between thermal and bulk Comptonization (TC and BC, respectively) using the BMC model in XSPEC. The relative contribution between the two Comptonization regimes (thermal versus bulk) is proposed to be drawn by the local accretion rate $\dot{M}$. Starting from the lowest level $\dot{M}$, the low/hard state spectra, whose cut-off is below $100 \mathrm{keV}$, can be explained by TC of soft photons by a hot $(\sim 30 \mathrm{keV})$ electron population; for increasing $\dot{M}$, bulk Comptonization becomes more relevant and moves to higher energies the cut-off ( $\mathrm{ard} / \mathrm{PL}$ state); higher accretion-rates produce a higher bulk-inflow Comptonization efficiency, which is evident in an extended hard tail in the spectrum above $30 \mathrm{keV}$ (intermediate state). In this state, TC becomes less efficient, since the coronal plasma is cooled to $\sim 3 \mathrm{keV}$, and the emergent spectrum is dominated by a strong thermal bump (with cut-off energy around $10 \mathrm{keV}$ ) that overlaps with a highenergy PL-like tail up to about $150 \mathrm{keV}$. The soft state spectra, with highest $\dot{M}$, are described by a single TC component (the "bump") with a low- $k T_{\mathrm{e}}$ and high- $\tau$ plasma, as expected in a high $\dot{M}$ environment. The emission above $30 \mathrm{keV}$ is no longer present since the high local-pressure gradient either prevents matter to reach the NS or strongly decelerates it ${ }^{1}$.

The study of the average spectra of the twelve NS LMXBs above $20 \mathrm{keV}$ allowed P06 to present a qualitative scenario for the X-ray spectral evolution of these sources along with the radio - X-ray connection. Nevertheless, the lack of data below $20 \mathrm{keV}$ prevented the authors from drawing more stringent conclusions about the accretion geometry of the systems and the physical parameters. To overcome these limitations and obtain a quantitative view of the parameter evolution for a single source, we present a study of the broad-band (0.4-120 keV) BeppoSAX spectra of Cyg X-2 with a newly updated version of the BMC model,

\footnotetext{
${ }^{1}$ See P06 for a more detailed description of the X-ray spectral evolution and for the X-ray/radio correlation obtained.
}

hereafter referred to as COMPTB (Farinelli et al. 2008, hereafter F08). A similar analysis of BeppoSAX, RXTE, and INTEGRAL data for a number of LMXBs was completed by Farinelli et al. (2007, hereafter F07) and F08.

\section{Observations and data analysis}

We analyzed two BeppoSAX (Boella et al. 1997a) observations of Cyg X-2, the first performed on 1996 July 23 (00:54:21 to 23:53:06 UT) and the second on 1997 October 26 (15:37:11 UT) to October $28(04: 00: 12$ UT). This data set was presented by $\mathrm{DS} 02$. In the analysis carried out by DS02, colour-colour and hardness intensity diagrams (CD and HID, respectively) of the source with the Medium-Energy Concentrator Spectrometer (MECS; Boella et al. 1997b), were produced. The soft colour (SC) was defined as the $4.5-7 \mathrm{keV} / 1.4-4.5 \mathrm{keV}$ count-rate ratio, while the hard colour was the $7-10.5 \mathrm{keV} / 4.5-7 \mathrm{keV}$ count-rate ratio. On the other hand, in the HID the intensity was defined by the $1.4-10.5 \mathrm{keV}$ count rate. During the 1996 observation, the source was in the HB of the CD/HID diagram; this is more evident using the HID, where two distinct regions separated by a gap around $150 \mathrm{cts}$ Sect. 1 are clearly visible (see Fig. 1 here, right hand panel, and Fig. 1 in DS02). These two HB regions were labeled as an upper HB and a lower HB (UHB and LHB, respectively) and two broad-band spectra were extracted separately for each of them. We adopted the same approach. On the other hand, during the 1997 observation, the source was in the $\mathrm{NB}$; in this case, the NB structure is more evident using the CD. Three spectra were extracted by DS02 for the NB, and labeled as an upper NB, a medium NB and a lower LNB (UNB, MNB and LNB, respectively, see Fig. 2, left panel, in DS02). We note however that the time-filters used by DS02 to extract the source spectra of the three parts of the NB were approximate because they considered three consecutive time intervals associated to the periods spent by the source on average at UNB, MNB and LNB stages, respectively. We realize however that this is not true as the source motion along the NB is not continuous and smooth but behaves randomly; this is clearly evident by plotting e.g., the $\mathrm{SC}$ as a function of time (see Fig. 2). To produce spectra corresponding to effective $\mathrm{UNB}, \mathrm{MNB}$, and LNB positions, we thus used a more refined criterion; we divided the NB in three intervals (see Fig. 1, left panel), depending on whether the SC-value was greater than 0.25 (UNB), between 0.23 and 0.25 (MNB) and lower than 0.23 (LNB), but temporal filter for spectral extraction 


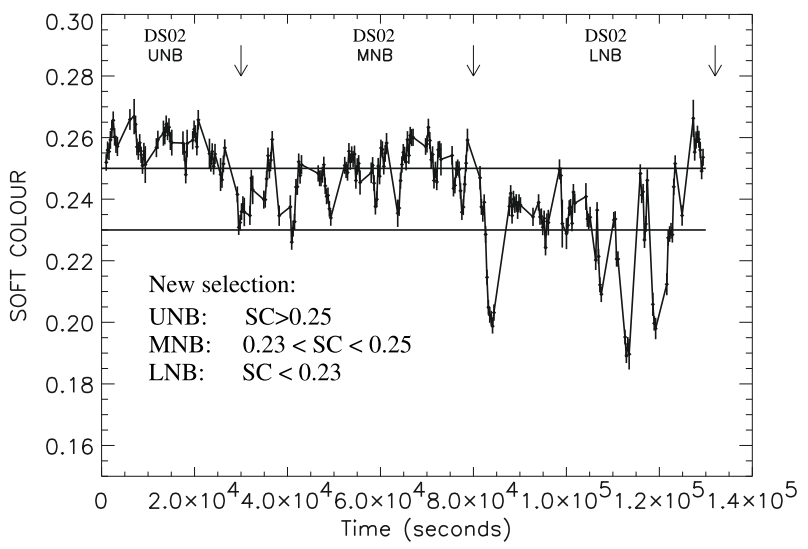

Fig. 2. Temporal evolution of Cyg X-2 soft colour in the NB. The three intervals used by DS02 to classify the NB are shown with the upper arrows. The soft-colour intervals used in this work are given and shown by the horizontal lines.

were produced following the source behaviour as reported in Fig. 2.

The shift in the source position within the $\mathrm{CD} / \mathrm{HID}$, between our plots and those reported in DS02, has two reasons: first, we extracted light curves (such as energy spectra) from a $4^{\prime}$ region centered on MECS source image (while in DS02 no spatial selection was used for producing CD/HID), and second we used a more precise MECS instrumental channel-to-energy conversion law, required when extracting light curves in different energy bands.

The time-filtered spectra of the high-energy Phowswich Detection System (PDS; Frontera et al. 1997) were produced using the XAS package, and we grouped the PDS channels to ensure that we had signal-to-noise $\gtrsim 3$ for each channel. Bins with lower data quality were discarded and not considered during fitting. A further comment is required when using the LECS response files (the energy redistribution matrix and the effective area files, RMF and ARF, respectively). When the LECS count rate is about 50-60 cts Sect. 1 or lower, the standard on line available response matrix can be safely used, while for higher count rates, specific observation-related RMF and ARF files are required. The fits to the LECS Crab spectrum ( 200 cts Sect. 1) clearly shows the presence of $\$ 5 \%$ residuals in the region 0.5 $1 \mathrm{keV}$ and $2-3 \mathrm{keV}^{2}$. It is thus evident that when a source spectrum is very bright and far from being Crab-like, these instrumental features can be significantly enhanced. This problem may be critical for LMXBs since their low-energy X-ray emission is dominated by the presence of the soft $\sim 0.5 \mathrm{keV}$ blackbody (BB) component in addition to photoelectric absorption. The LECS count rate of the 1996 observation (HB spectra) of Cyg X-2 was around 50 cts Sect. 1 ( $\sim .25$ Crab), while during 1997 observation (NB spectra) it was on average $\sim 90$ cts Sect. 1 ( $\sim 0.4 \mathrm{Crab})$. We thus produced new RMF and ARF files for each of the five selected spectra using the LEMAT package. A fit to the Crab LECS spectrum shows that $0.8 \%$ of systematic error plus a fake $2.2 \mathrm{keV}$ Gaussian emission line provide a reduced $\chi^{2}$ equal to 1 . The fake excess around $2.2 \mathrm{keV}$ is observed not only in LECS but also in MECS spectra, and is the result of a strong edge-like decrease in the instrument effective area for both instruments, which becomes evident at very high instrument count rates; this excess is observed in all our five spectra. While considering the instrumental issues mentioned above,

\footnotetext{
2 See http://bepposax.gsfc.nasa.gov/bepposax/software/ index.html
}

we added a $0.5 \%$ systematic error to all instruments; this is a good compromise between taking into account calibration uncertainties and avoiding unreasonably low reduced $\chi^{2}$-values in the best-fit models. We also added a Gaussian emission line to the critical edge region ( $2.2 \mathrm{keV})$ of LECS and MECS. Another issue at low energies concerns the strong feature observed in the Cyg X-2 LECS spectra (especially in the 1997 data set) around $1 \mathrm{keV}$. Since its first detection during a rocket flight in 1971 (Bleeker et al. 1972), several experiments have reported this emission. While from the Ariel $V$ data (Branduardi-Raymont et al. 1984) it was impossible to estimate the line equivalent width (EW) because of the low instrumental sensitivity, using Einstein observations Vrtilek et al. (1988) suggested that this feature was likely due to a combination of unresolved Fe L-shell line emission that they identified with Fe XVII, O VIII/Fe XVII, $\mathrm{Fe} \mathrm{XX} / \mathrm{Ni} \mathrm{XX}$ and Fe XVII/Fe XXII-XXIV features ranging from 0.74 to $1.12 \mathrm{keV}$. Analysis of EXOSAT observations also supported this hypothesis (Chiappetti et al. 1990), although the data did not allow this complex region to be resolved. Based on subsequent measurements with $B B X R T$ and $A S C A$, Smale et al. (1993, 1994) modeled the excess with a broad Gaussian line of full width half-maximum and EW in the range $\sim 0.05-0.33 \mathrm{keV}$ and $8-60 \mathrm{eV}$, respectively. This modeling also provided a good description of the excess in BeppoSAX observations (Kuulkers et al. 1997), resulting in an energy centroid of $1 \mathrm{keV}$, with $\sigma \sim 0.5 \mathrm{keV}$ and $\mathrm{EW} \sim 70 \mathrm{eV}$. By assuming the model proposed by Vrtilek et al. (1988), Kuulkers and collaborators found similar results but measured difference in EW, confirming that the shape of the feature, as well as its EW, changes from observation to observation. However, we note that both Chandra (Takei et al. 2002) and XMM-Newton (Costantini et al. 2005) observations of $\mathrm{Cyg} \mathrm{X}-2$ revealed a complex structure of absorption edges (due to a combination of $\mathrm{N}, \mathrm{O}, \mathrm{Fe}-\mathrm{L}$, and $\mathrm{Ne}-\mathrm{K}$ shells) in the region $0.4-0.8 \mathrm{keV}$ but no emission around $1 \mathrm{keV}$. Due to the high-energy resolution of these two satellites at low energy and since the occasional presence of a strong absorption edge, if not taken into account, may produce emission-like features in the residuals, we tried to use a single absorption edge in our best-fit models; the result was however unsatisfactory, since it required a Gaussian emission line. Investigating the origin (physical or instrumental) of this $1 \mathrm{keV}$ emission feature is beyond the scope of the present paper and all results reported include a Gaussian emission line. Independently from the physical or instrumental origin of the $1 \mathrm{keV}$ emission line, we note that its presences strongly influences the determination of both the interstellar absorption $N_{\mathrm{H}}$ and the temperature of the soft BB-like component. In particular, we find that too low $N_{\mathrm{H}}$-values are obtained for the latter parameter $\left(\sim 0.08 \times 10^{22} \mathrm{~cm}^{-2}\right)$ if compared to results of radio map measurement $\left(\sim 0.19 \times 10^{22} \mathrm{~cm}^{-2}\right.$; Dickey \& Lockman 1990), XMM observations $\left(\sim 0.18 \times 10^{22} \mathrm{~cm}^{-2}\right.$; Costantini et al. 2005), or BeppoSAX observation with no line included $\left(\sim 0.25 \times 10^{22} \mathrm{~cm}^{-2} ;\right.$ DSO2, F08). The differences in $N_{\mathrm{H}}$ are more significant for the NB spectra than the HB ones: we thus fixed $N_{\mathrm{H}}=0.15 \times 10^{22} \mathrm{~cm}^{-2}$ for all five analyzed spectra, to ensure that these model-dependent variations were as low as possible.

\section{Results}

The overall continuum of the five spectra was studied using COMPTB $^{3}$, the newly developed Comptonization model

\footnotetext{
3 The COMPTB model is freely available at web site http:// heasarc.gsfc.nasa.gov/docs/xanadu/xspec/newmodels.html
} 
Table 1. Best-fit parameters of the multi-component model WABS(COMPTB + COMPTB + Gaussian).

\begin{tabular}{|c|c|c|c|c|c|}
\hline Parameter & $U H B$ & $L H B$ & $U N B$ & $M N B$ & $L N B$ \\
\hline$N_{\mathrm{H}}^{a}$ & {$[0.15]$} & {$[0.15]$} & {$[0.15]$} & {$[0.15]$} & {$[0.15]$} \\
\hline \multicolumn{6}{|c|}{ COMPTB (thermal: $\log [\mathrm{A}]=8, \delta=0$ ) } \\
\hline$k T_{\mathrm{s}}(\mathrm{keV})$ & $0.28_{-0.03}^{+0.02}$ & $0.29_{-0.02}^{+0.02}$ & $0.31_{-0.01}^{+0.01}$ & $0.30_{-0.01}^{+0.01}$ & $0.31_{-0.01}^{+0.01}$ \\
\hline$k T_{\mathrm{e}}(\mathrm{keV})$ & $2.72_{-0.08}^{-0.03}$ & $2.65_{-0.10}^{+0.02}$ & $2.41_{-0.04}^{-0.01}$ & $2.23_{-0.05}^{+0.01}$ & $2.51_{-0.08}^{+0.01}$ \\
\hline$\alpha$ & $0.80_{-0.03}^{+0.03}$ & $0.82_{-0.03}^{+0.07}$ & $0.80_{-0.03}^{+0.04}$ & $0.82_{-0.04}^{-0.05}$ & $1.00_{-0.02}^{+0.08}$ \\
\hline$\tau^{b}$ & $6.5^{-0.05}$ & $6.4^{-0.03}$ & $6.9^{-0.05}$ & $7.1^{-0.04}$ & $5.8^{-0.02}$ \\
\hline $\mathrm{CAF}^{c}$ & 2.8 & 2.6 & 2.5 & 2.4 & 2.2 \\
\hline \multicolumn{6}{|c|}{ СОМРTB } \\
\hline$k T_{\mathrm{s}}(\mathrm{keV})$ & $1.00_{-011}^{+0.08}$ & $1.15_{-0.14}^{+0.05}$ & $1.11_{-0.02}^{+0.02}$ & $1.08_{-0.03}^{+0.03}$ & $1.16_{-0.04}^{+0.03}$ \\
\hline$R_{\mathrm{bb}}^{d}(\mathrm{~km})$ & $3.4^{-0.11}$ & 3.2 & $4.6^{-0.02}$ & $4.9^{-0.05}$ & $4.6^{-0.04}$ \\
\hline$k T_{\mathrm{e}}(\mathrm{keV})$ & {$\left[k T_{\mathrm{e}}\right]$} & {$\left[k T_{\mathrm{e}}\right]$} & {$\left[k T_{\mathrm{e}}\right]$} & {$\left[k T_{\mathrm{e}}\right]$} & - \\
\hline $\log (A)$ & $0.11(>-0.12)$ & $-0.40(>-0.65)$ & {$[-0.4]$} & {$[-0.4]$} & {$[-8]$} \\
\hline$\alpha$ & {$[2.5]$} & {$[2.5]$} & {$[2.5]$} & {$[2.5]$} & - \\
\hline$\delta$ & $75(>40)$ & $49_{-18}^{+39}$ & $25_{-4}^{+5}$ & $22_{-4}^{+5}$ & - \\
\hline \multicolumn{6}{|c|}{ Gaussian } \\
\hline$E_{l}(\mathrm{keV})$ & $6.8_{-0.1}^{+0.1}$ & $6.7_{-0.1}^{+0.1}$ & $6.7_{-0.1}^{+0.1}$ & $6.6_{-0.1}^{+0.1}$ & $6.7_{-0.1}^{+0.1}$ \\
\hline$\sigma_{l}(\mathrm{keV})$ & $0.3_{-0.1}^{+0.1}$ & $0.2(<0.3)$ & $0.1(<0.2)$ & $0.12(<0.2)$ & $0.1 \quad(<0.2)$ \\
\hline$I_{l}$ & $2.5_{-0.9}^{+0.1}$ & $1.4_{-0.6}^{+0.7}$ & $1.9_{-0.7}^{+0.7}$ & $1.8_{-0.8}^{+0.9}$ & $3.0_{-0.9}^{+1.7}$ \\
\hline$E W_{l}(\mathrm{eV})$ & $38_{-9}^{+18}$ & $17_{-8}^{+8.0}$ & $15_{-5}^{+5}$ & $14_{-6}^{+7.8}$ & $29_{-9}^{+16}$ \\
\hline$L_{\mathrm{bb}}^{e} / L_{\mathrm{tot}}$ & 0.25 & 0.26 & 0.25 & 0.25 & 0.28 \\
\hline$L_{\mathrm{th}}^{f} / L_{\mathrm{tot}}$ & 0.69 & 0.67 & 0.63 & 0.61 & 0.63 \\
\hline$L_{\text {tot }}^{\mathrm{th}^{\prime}}$ & 0.9 & 1.1 & 1.7 & 1.7 & 1.5 \\
\hline$\chi^{2} /$ d.o.f. & $175 / 152$ & $162 / 154$ & $159 / 148$ & $185 / 144$ & $197 / 143$ \\
\hline
\end{tabular}

Errors are computed at $90 \%$ confidence level for a single parameter. Additional emission lines at $\sim 1 \mathrm{keV}$ and $\sim 2.5 \mathrm{keV}$ were included in all the five spectra (see Sect. 2). ${ }^{a}$ In units of $10^{22} \mathrm{~cm}^{-2} ;{ }^{b}$ computed from $k T_{\mathrm{e}}$ and $\alpha$ using Eqs. [17] and [24] for slab geometry in Titarchuk \& Lyubarskij (1995); ${ }^{c}$ Compton Amplification Factor (see text for the definition); ${ }^{d}$ computed using the simple relation $L=4 \pi \sigma R^{2} T^{4} ;{ }^{e}$ ratio between the direct BB component flux of the second СОМРTB and the total flux in the $0.1-200 \mathrm{keV}$ energy range; ${ }^{f}$ ratio between the TC component flux and the total flux in the $0.1-200 \mathrm{keV}$ energy range; ${ }^{g}$ in units of $10^{38} \mathrm{erg} \mathrm{s}^{-1}$ computed in the energy range $0.1-200 \mathrm{keV}$ assuming a source distance of $8 \mathrm{kpc}$.

described in detail by F08. We note that while F08 selected different sources in a given spectral state, for this present work we selected a single source and studied its spectral evolution, from its intermediate state to its soft state (following the terminology of P06), to compare the model parameter evolution during the spectral transition. We remind the reader that $\mathrm{Z}$ sources, unlike atolls, have never been detected in the low/hard or $h a r d / P L$ state, possibly because they never reach sufficiently low accretion rate levels. Thus, the intermediate state and the spectral transition to the soft state is a complete pattern of the spectral evolution for a $\mathrm{Z}$ source.

We recall the main characteristics of the COMPTB model: the total emerging spectrum is given by

$F(E)=\frac{C_{N}}{1+A}(B B+A \times B B * G)$,

where the first and second terms of the right-hand side represent the seed BB-like photon spectrum and its convolution with the system Green's function (Comptonized spectrum), respectively. The factor $1 /(1+A)$ is the fraction of the seed-photon radiation as seen directly by the Earth observer, whereas the factor $A /(1+A)$ is the fraction of the seed-photon radiation upscattered by the Compton cloud. Free parameters of the model are the BB seed-photon colour-temperature $k T_{\mathrm{s}}$ and normalization $C_{\mathrm{N}}$, the plasma temperature, $k T_{\mathrm{e}}$, and the logarithm of the illuminating factor $A, \log (A)$. The information about the efficiency of the Comptonization is given by two parameters, $\alpha$ and $\delta$. The $\alpha$-parameter (photon index $\Gamma=\alpha+1$ ) indicates an overall Comptonization efficiency related to an observable quantity in the photon spectrum of the data. The lower the $\alpha$ parameter (spectrum extending to higher energies) the higher the efficiency, i.e., the higher energy transfer from hot electrons to soft seed photons. The $\delta$-parameter provides information about the efficiency of $\mathrm{BC}$ with respect to that of TC. Both $\alpha$ and $\delta$ are closely related (see Fig. 1 in F08) and only in the case of a clear cut-off in the hard X-ray tail data a precise estimate of the efficiency of both effects is possible.

Each continuum in the five spectra of Cyg X-2 (two HB and three NB) was fitted using the sum of two COMPTB components. In Table 1, we report the results of our spectral analysis. In all cases, the first COMPTB component (pure TC, $\delta=0$ ) significantly contributes to the total emerging spectrum. Most of the source energetic budget (60-70\%) is, in fact, determined by this component which is described (a well-known feature of Z-class LMXBs) by Comptonization of $\sim 0.5 \mathrm{keV}$ photons by cool electrons $\left(k T_{\mathrm{e}} \sim 3-5 \mathrm{keV}\right)$ in a high optical-depth environment ( $\tau \sim 5-10$ depending on the geometry of the cloud, slab or sphere, respectively). The direct seed BB-like spectrum is not seen in this case because of the high optical thickness of the Compton cloud. We note that the BB temperature at UHB stage is a factor of about two higher than the value reported by F08 for the same data set. This is because we included a Gaussian emission line at $1 \mathrm{keV}$, which, as already stated in Sect. 2, may affect both the estimated interstellar absorption $N_{\mathrm{H}}$ and the parameters of low-energy continuum features.

In addition to dominant TC component, broad-band spectra of high-luminosity LMXBs also exhibit a $\sim 1 \mathrm{keV}$ BB-like component, whose origin is generally claimed to be close to 


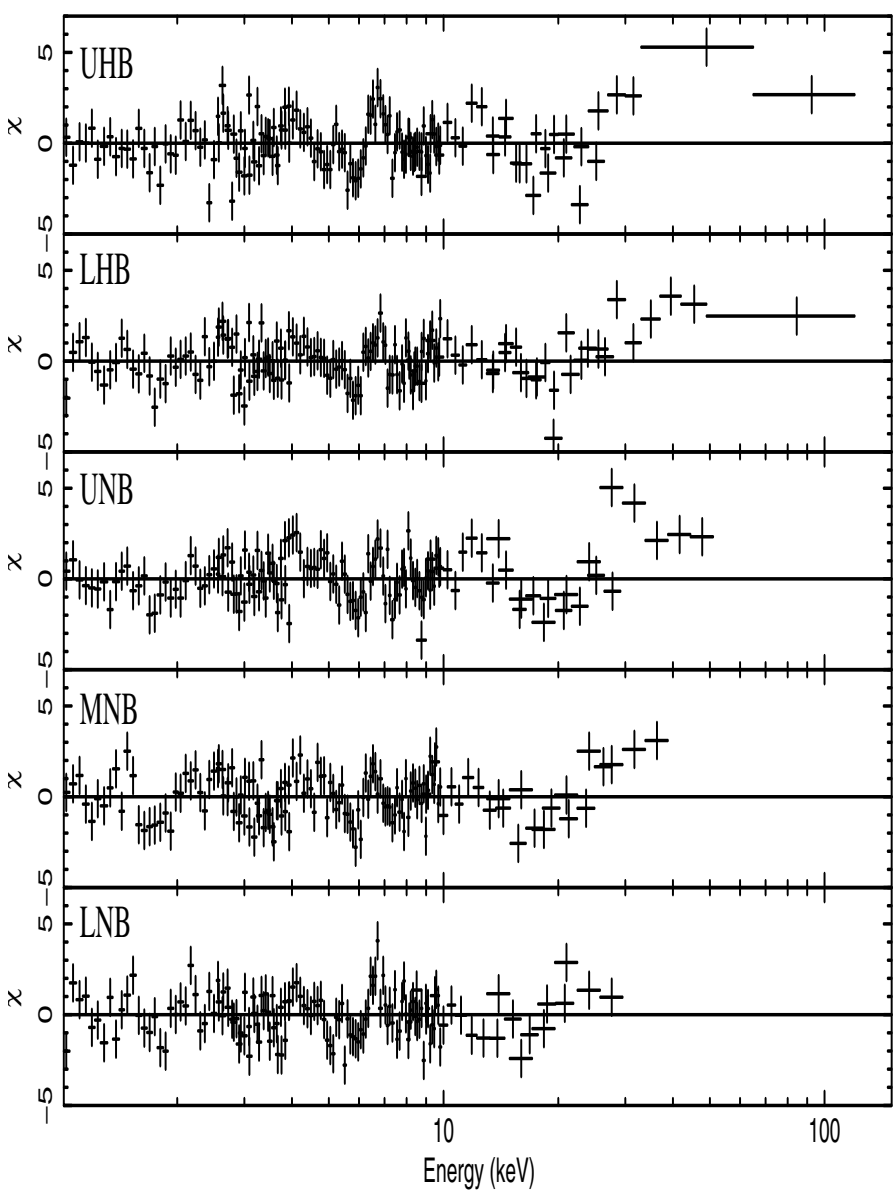

Fig. 3. Residuals in units of $\sigma$ between the data and best-fit model consisting of a pure TC spectrum (COMPTB with $A \gg 1$ and $\delta=0$ ) and a simple BB (the latter obtained as COMPTB with $A \ll 1$ ). The Gaussian emission lines at $\sim 1$ and $\sim 2.5 \mathrm{keV}$ are also included. The high-energy part $(>20 \mathrm{keV})$ of the spectrum can be described by simple TC component only at LNB (lower panel) but the fit gets progressively worse up towards UHB where systematic deviation of the data is clearly visible.

the NS surface. This low-energy component is included in our spectra with a second COMPTB model, which, in fact, takes into account the presence of a directly visible BB-like spectrum (see Eq. (1)). In all five spectra, there is also an evidence of a Gaussian emission line around $6.7 \mathrm{keV}$. We note, however, that this continuum model (TC plus BB) provides accurate description of the data only for the spectrum at the LNB stage, while a systematic excess above $\gtrsim 20 \mathrm{keV}$ is observed mainly at UHB and LHB with decreasing strength in this excess at UNB and MNB (see Fig. 3). This systematic deviation of data from the typical soft-state continuum model is, in fact, a well-known example of high-energy transient behaviour of some LMXBs, including Cyg X-2 (see also DS02).

This high-energy feature can in general be fitted by a simple PL, but for a more physical approach to X-ray spectral formation, one can consider the feature to be due to $\mathrm{BC}$ emission (see the second term in Eq. (1)). The latter process describes the result of Comptonization of a seed-photon population by a medium subjected to inward bulk motion, such that both TC and $\mathrm{BC}$ contribute to the emergent spectrum. It is then necessary to introduce additional free parameters to describe the physical properties of the bulk region, namely $\alpha, \delta, k T_{\mathrm{e}}$, and $\log (A)$.

However, if the number of free parameters describing the high-energy part of the X-ray spectrum (i.e., the second

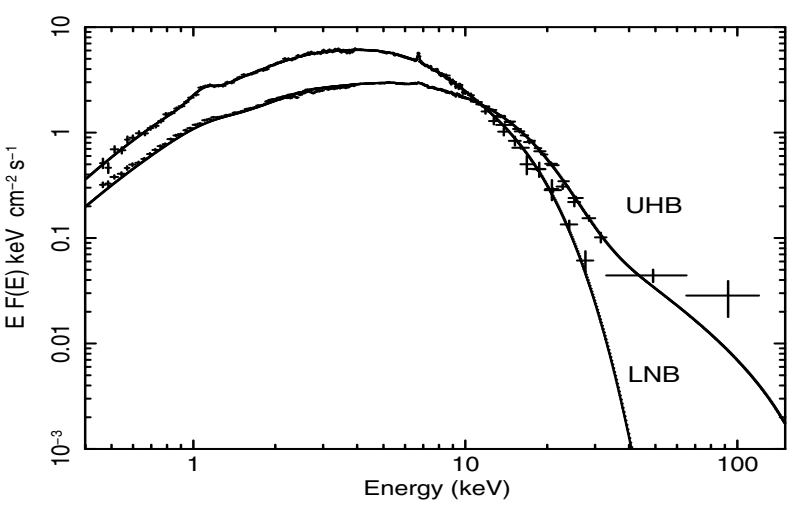

Fig. 4. Unabsorbed $\mathrm{EF}(\mathrm{E})$ spectra and best-fit models (see Table 1) of the UHB and LNB spectra of Cyg X-2, which show the spectral evolution of the source from its intermediate state (UHB) to the high-soft state (LNB).

COMPTB in Table 1) exceeds the number of observable quantities (PL slope, normalization, and high-energy spectral cut-off, if observed), it becomes impossible to constrain the parameter values and assumptions are required to reduce the dimension of the free-parameter space. The first of our assumptions is that the Comptonizing plasma temperatures of the two COMPTB models are equal each other (see Table 1). As already pointed out by F08, significant variation in electron temperature is unexpected in the region extending from the outer TC medium to the innermost bulk region. The lack of an unambiguous rollover $(\gtrsim 100 \mathrm{keV})$ in the high energy data reduces the number of measurable spectral characteristics in the high-energy transient X-ray emission, which are essentially the PL slope and its normalization. The latter quantity is described by $\log (A)$ in the model, while the spectral index $\alpha$ provides the spectral slope. For a given fixed value of $\alpha$, when the bulk parameter $\delta$ increases, the cut-off energy also increases, leading to the extension of the PL-like component in the spectrum (see Fig. 1 in F08).

The statistics of the spectral data at UHB and LHB stages did not allow us to simultaneously constrain $\alpha, \log (A)$, and $\delta$. Since we are interested mainly in studying the importance of $\mathrm{BC}$ as a function of the source position in the $\mathrm{Z}$ track, this mapping was possible only allowing the bulk parameter $\delta$ to be a free parameter and the energy index $\alpha$ to be fixed. A detailed study of the parameter space showed us that the minimum in the $\chi^{2}$ could be reached by fixing $\alpha=2.5$. The results obtained with this modeling approach are reported in Table 1. One can see only that the lower limit (at 90\% confidence level) on $\log (A)$ can be determined in both HB spectra, while as far as $\delta$ is concerned, we have a lower limit to its value at UHB and a stronger constrain of its value at LHB. The situation becomes even more critical for the parameter determination of the NB spectra. In the UNB and MNB cases, where a high-energy excess is observed above the BB plus TC continuum (see Fig. 3), it was impossible to place strong constraints on both $\delta$ and $\log (A)$ by allowing them to be simultaneously free, mainly because of the poor quality of the data at high energies. The only possibility for exploring the behaviour of $\delta$ was thus fixing $\log (A)$ to the best-fit value of the LHB spectrum (see Table 1). Finally, in the LNB spectrum the second COMPTB model requires neither TC nor BC, resulting in a simple BB shape. The overall LNB spectrum could hence be well reproduced by the sum of TC (the first COMPTB) plus a BB. In terms of improving the quality of the fit, the addition 
of a TC plus BC component to the simple BB gives $\Delta \chi^{2} \sim 100$ (F-test $\sim 10^{-17}$ ) and $\Delta \chi^{2} \sim 63$ (F-test $\sim 10^{-10}$ ) for the UNB and MNB spectra, respectively, while for the LNB spectrum it is only $\Delta \chi^{2} \sim 19$ (F-test $\left.\sim 10^{-4}\right)$.

\section{Discussion}

We have studied newly extracted Cyg X-2 BeppoSAX spectra, covering the $\mathrm{HB}$ and $\mathrm{NB}$ of the $\mathrm{Z}$ track, using the COMPTB model developed by Farinelli et al. (2008). We remark that COMPTB is a Comptonization model including both $\mathrm{TC}$ and $\mathrm{BC}$, i.e., that can be considered as an (updated) merging of the well known COMPTT and BMC XSPEC models.

\subsection{Interpretation of the observed spectra}

We have fitted the continuum of five BeppoSAX average Cyg X-2 spectra with two COMPTB models. The first one represents the dominating TC component of the spectrum, while the second model describes the strong $\sim 1 \mathrm{keV}$ BB-like feature and, when observed, the hard X-ray emission above $30 \mathrm{keV}$. In our scenario (see also F07 and F08), the thermal COMPTB describes emission that originates in the relatively cold $(\sim 3 \mathrm{keV})$ and optically thick $(\tau \sim 5)$ outer transition layer due to the Comptonization of cold, disk seed-photons $(\sim 0.3 \mathrm{keV})$. This component is rather stable as can be seen in Table 1 . The second СОМРТВ component originates in the innermost region of the system and experiences significant change (see Table 1). Hot BB photons $(\sim 1 \mathrm{keV})$ are emitted by the neutron-star surface and within the transition layer itself. The spectral shape variability is due to the changing Comptonization of this seed-photon population, which is determined mainly by the accretion rate, as discussed in Sect. 4.2. The difference in the measured values of $k T_{\mathrm{s}}$ of a factor about three is strongly indicative of two distinct populations of seed photons, which would translate in the existence of two physically separated regions. By studying the bestfit parameters in Table 1, the source spectral stability of the persistent continuum is almost evident, in general agreement with high-luminosity LMXBs properties. In the MNB and LNB spectra, the TC parameters are also well constrained, which differs from the results reported by DS02, where they significantly deviate from a smooth trend with large associated errors. We claim that one reason for the DS02 results could be the approximate timing selection for spectral analysis (see Sect. 2). The quantity that undergoes the most significant change as a function of source position in the CD/HID is the estimated $0.1-200 \mathrm{keV} \mathrm{lu}-$ minosity, which has a maximum increase of about $80 \%$ between the UHB and UNB/MNB state. Less significant variations are observed in the plasma temperature $k T_{\mathrm{e}}$ and the thermal component Comptonization amplification factor (CAF), defined to be the ratio of Comptonized photon energy flux to that of seed photons. The CAF decreases monotonically from the UHB to the LNB. We also observe that the inferred plasma temperature, optical depth, and luminosity in the LNB deviates slightly from the monotonic trend observed in the first four spectra. Whether this small jump is real or due to some bias in the spectral modeling is not easy to determinate. In particular, the presence of a strong $1 \mathrm{keV}$ and a weaker $2.5 \mathrm{keV}$ emission feature especially in the NB spectra may affect the true determination of the seedphoton temperature of the pure thermal COMPTB model, which is constrained by the $k T_{\mathrm{e}}$ and $\alpha$-values.

\subsection{Spectral evolution as a function of mass accretion rate}

The theoretical and observational support provided in the past years to the study of hard X-ray emission in LMXBs (e.g., Titarchuk et al. 1996, 1997; P06; F07; F08) has advanced significantly our knowledge of the emission-process nature in these sources. Multi-wavelength observations of Z sources (e.g., Penninx et al. 1988; Vrtilek et al. 1990) strengthened the evidence that the motion of the sources along the $\mathrm{Z}$ track is controlled mainly by the accretion rate $\dot{M}$, which increases between the HB and the FB. However, little is said on why an increase in $\dot{M}$ leads to the disappearance of the hard tail in LMXBs and the true meaning of $\dot{M}$ itself is not fully understood. It is however widely accepted that the simple source bolometric X-ray luminosity is not a good $\dot{M}$-tracer, for NS or also black hole (BH) systems (e.g., van der Klis 2001). We propose to define two $\dot{M}$-values for a single source, one, $\dot{M}_{\text {disk, }}$, related to the accretion disk and another, $\dot{M}_{\mathrm{tl}}$, to the so-called transition layer (TL), the region where the disk angular velocity deviates from Keplerian rotation and adjusts to the angular velocity of the slowly spinning NS. The reason for this split is that at very high accretionrate levels (close to the Eddington limit), the radiation pressure from the accretion disk can eject a significant fraction of the accreted matter, producing a powerful wind surrounding the system (Bradshaw et al. 1997). In this case the mass flow originating in the innermost part of the disk to the TL and then the NS surface is less than that arriving at the disk outer part, namely $\dot{M}_{\mathrm{tl}} \lesssim \dot{M}_{\text {disk}}$, with $\dot{M}_{\mathrm{tl}} \propto \dot{M}_{\text {disk }}$ (Titarchuk et al. 2007b).

Focusing the attention on the TL region, we note that both theoretical and extended data-analysis works (see references above) have proposed that the bulk Comptonization process in the TL is responsible for the hard X-ray emission in NS LMXBs. A self-consistent physical treatment of the TL region is presently being developed by Titarchuk \& Farinelli (2009, in preparation, hereafter TF09). The numerical solution of the radial momentum equation performed by TF09 unambiguously demonstrate that matter arriving at the transition radius from the Keplerian disk with some fraction of the characteristic disk radial (magnetoacoustic) velocity then proceeds through the transition layer towards the NS with almost constant velocity up to some radius at which it proceeds further in quasi free-fall manner, reaching the NS surface with about $10 \%$ of the speed of light. However, as TF09 emphasize, this free-fall region is not always present but depends on the mass accretion rate $\dot{M}$, which can thus be now identified with $\dot{M}_{\mathrm{tl}}$. In particular, high $\dot{M}_{\mathrm{tl}}$ generates a strong radiation field in the TL, whose pressure gradient prevents matter from reaching the NS surface with the high speed necessary to produce, via a Doppler effect, hard X-ray photons (hence no hard tail detected). We recall again that the only observable quantities in the high-energy X-ray spectra are the PL photon index $\gamma=\alpha+1$, its normalization, and the high-energy cut-off, if detected. The true accretion rate (regardless of its definition) is not a directly measurable quantity; it can be estimated only including it as a free parameter in dedicated accretion models. This is, e.g., the approach followed by Becker \& Wolff (2007) in their model of accretion columns in X-ray pulsars. On the other hand, in СОМРТВ the accretion rate is not a free parameter, but is implicitly hosted in the bulk parameter as $\delta \propto \dot{m}^{-1}$ (where $\dot{m} \equiv \dot{M}\left(\dot{M}_{\mathrm{Edd}}\right)$. We also note that in the intermediate state spectra of NS LMXBs, two spectral indexes are observed in the X-ray spectrum, the first one related to pure TC and the second to a mixed thermal-bulk Comptonization effect.

For Cyg X-2, the TC spectral index $\alpha$ is about 1 (see Table 1), which is a typical value of the intermediate state in 

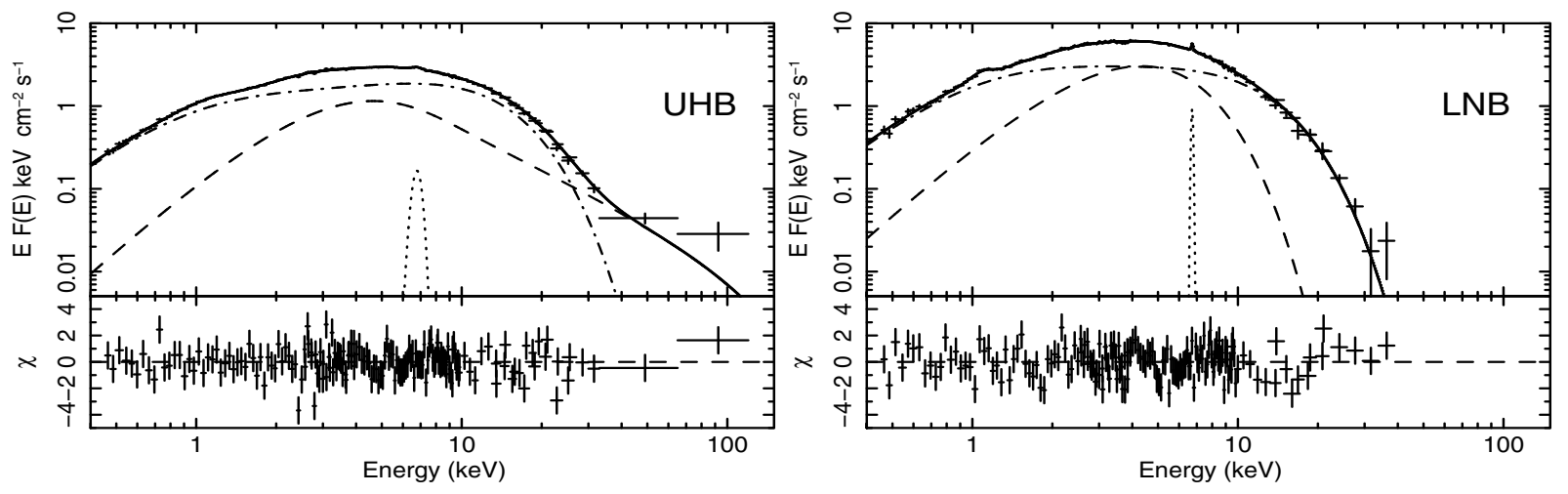

Fig. 5. Unabsorbed $\mathrm{EF}(\mathrm{E})$ spectra, best-fit model and residuals between data and model in units of $\sigma$ for the UHB and LNB spectra of Cyg X-2. Different line styles represent single component of the best-fit model reported in Table 1. Dotted-dashed: CoMPTB with pure TC. Long-dashed: СОМРТВ which includes TC and BC, i.e. $\delta \gg 1$ (left panel) or with simple BB spectrum (right panel). Dotted: Gaussian emission line.

NS sources ${ }^{4}$. As far as the second spectral index is concerned, it is important to discuss the differences between systems hosting a NS or a BH. For BH sources, affected by enhanced spectral transitions, in general only one index is measured from the spectrum. It evolves from $\alpha \sim 1.7$ in the hard state, which is TC-dominated and with observed cut-off at about $100 \mathrm{keV}$, to $\alpha \sim 2.8$ in the BCdominated soft state. In this case, index saturation is observed (Shaposhnikov \& Titarchuk 2007). It can be proven (Bradshaw et al. 1997) that $\alpha \approx Y^{-1}$, where the Comptonization parameter $Y=\eta N_{\mathrm{sc}}, \eta$ is the average energy exchange per scattering, and $N_{\mathrm{sc}}$ is the average number of scatterings. In a BC-dominated flow, $\eta \propto \tau^{-1}$ and $N_{\text {sc }} \propto \tau$ (Laurent \& Titarchuk 2007), so that saturation occurs. However this occurs in BH systems, where the presence of a full absorption inner boundary-condition for the radiation (Titarchuk et al. 1997) allows bulk motion always to be present. In the case of NS system, the presence of a solid surface (reflection boundary condition) plays a crucial role, given that the radiation pressure gradient increases with the accretion rate until the bulk flow stops. Thus, no index saturation can be observed in NS sources. The innermost part of the system evolves towards thermal equilibrium when the emergent spectrum consists of two BB-like components related to NS surface and disk emission (Titarchuk \& Shaposhnikov 2005).

On the basis of these considerations, we may tentatively discuss the parameters behaviour reported in Table 1: along the horizontal branch (HB), at a lower $\dot{M}$ level, the BC effect can be clearly detected, as is evident from the high $\delta$-value. A slight decrease in the BC effect is observed in the LHB spectra, where both $\delta$ and $\log (\mathrm{A})$ become lower, even though this effect seems to be marginal given the high error bars of both these parameters. The total (0.1-200 keV) source luminosity at LHB increases by about $20 \%$ with respect to that at UHB. When the sources is at the UNB (upper normal branch), a further increase ( 50\%) in the luminosity with respect to that at LHB occurs, accompanied by a significant decrease in the hard X-ray emission (see Fig. 3). We may interpret this behaviour as an increase in $\dot{M}_{\text {disk }}$ which can produce a higher $\dot{M}_{\mathrm{tl}}$ and consequently leads to an increase in the radiation pressure in the TL. This pressure works against an inward matter motion and the bulk effect decreases. Figure 4 illustrates this spectral transition, due a change in $\dot{M}$ from the intermediate (UHB) to soft (NB) state, following the terminology defined in P06.

\footnotetext{
4 This result can be verified from the best-fit values of $k T_{\mathrm{e}}$ and $\tau$ obtained from COMPTT in previous works (see references in Sect. 1) and using Eqs. [17] and [24] in Titarchuk \& Lyubarskij (1995).
}

In the spectrum, the PL extension reduces as a result of the $\mathrm{BC}$ efficiency ( $\delta$-parameter) reduction. Looking at the parameters in Table 1 and the residuals in Fig. 3, it is possible to see that the situation changes only slightly in the MNB. Finally, in the LNB no hard X-ray emission is observed and a simple BBlike spectrum along with the TC bump is present. It is possible that at this stage the effect of radiation pressure in the TL is strong enough to stop bulk motion, or at least bring its visible effect below the instrument threshold. At this stage, it is likely that matter follows a complex behaviour; it may arrive at the NS surface slowly or may spread significantly over the stellar surface (Inogamov \& Sunyaev 1999) producing additionally a thick layer that suppresses the radio emission (P06).

We note however that the $0.1-200 \mathrm{keV}$ luminosity at LNB is about $12 \%$ lower than that at UNB and MNB. These results clearly demonstrate that the X-ray luminosity is not a good tracer of the accretion rate, regardless of its precise definition. In all five spectra, a direct BB-like component, providing about $25 \%$ of the source energetic budget, is observed (indeed in the second COMPTB component, $\log (\mathrm{A})$ is small in all cases). The estimated $\mathrm{BB}$ colour radius in all cases is $R_{\mathrm{bb}} \lesssim 5 \mathrm{~km}$; while this value must be considered carefully (it is derived from the measured $\mathrm{BB}$ colour temperature $k T_{\mathrm{S}}$ assuming isotropic emission) its order of magnitude is consistent with the NS radius, pointing to an origin close to the compact object surface. In our interpretation, these BB-like seed-photon spectra have origins related to the thermal emissions of the NS surface and to local energyrelease emission in the TL region. It is possible that part of this soft component is direct radiation from the seed-photon region (NS or TL) and part consists of photons that are unaffected by the Comptonization. The fraction of the directly escaping radiation is parameterized by $\log (\mathrm{A})$, related to both a geometrical (covering factor) and spatial (seed photon distribution) system configuration in the innermost part, even though the relative importance among the two effects cannot be determined from the X-ray spectrum.

\section{Conclusions}

The scenario where both thermal and bulk Comptonization contribute to explaining the LMXB spectral evolution is gaining strong theoretical (Titarchuk et al. 1996, 1997; Laurent \& Titarchuk 1999, 2001) and observational support (Shrader \& Titarchuk 1998; Borozdin et al. 1999; P06; F07; F08; this work). This is also true in the case of accretion-powered X-ray pulsars for which a theoretical model based on thermal and bulk 
Comptonization occurring in accreting shocked gas was developed by Becker \& Wolff (2007). In their model the authors found that the effects of the strong magnetic field $\left(B \sim 10^{12} \mathrm{G}\right)$ become important and should be included, whereas in this paper we have used a model proposed by F08 to study low-magnetized compact objects ( $B \lesssim 10^{9} \mathrm{G}$ ), where effects of the magnetic field within the flow can be neglected (as opposed to the radiation pressure). The two models are complementary and we believe they can be an important step in understanding and relating the physics of bursting (low- $B$ ) and pulsating (high- $B$ ) accreting NS systems. Nevertheless, the present analysis has revealed the key importance of having a next generation of high-sensitivity instrument missions at energies extending up to $300-400 \mathrm{keV}$. The detection of high-energy cut-off in extended PL-like tails of both NS and $\mathrm{BH}$ binary systems would be one breakthrough in understanding the physics of accretion processes close to the compact object, providing constraints on the bulk energy of the matter.

Acknowledgements. The authors are very grateful to the anonymous referee, whose suggestions strongly improved the quality of the paper with respect to the formerly submitted version. A.P. acknowledges the Italian Space Agency financial and programmatic support via contract I/008/07/0. This work has been partially supported by the grant from Italian PRIN-INAF 2007, "Bulk motion Comptonization models in X-ray Binaries: from phenomenology to physics", PI M. Cocchi.

\section{References}

Asai, K., Dotani, T., Mitsuda, K., et al. 1994, PASJ, 46, 479

Barret, D. 2001, Adv. Space Res., 28, 307

Becker, P. A., \& Wolff, M. T. 2007, ApJ, 654, 435

Bleeker, J. A. M., Deerenberg, A. J. M., Yamashita, K., et al. 1972, ApJ, 178, 377

Boella, G., Butler, R. C., Perola, G. C., et al. 1997a, A\&AS, 122, 299

Boella, G., Chiappetti, L., Conti, G., et al. 1997b, A\&AS, 122, 327

Borozdin, K., Revnivtsev, M., Trudolyubov, S., Shrader, C., \& Titarchuk, L. 1999, ApJ, 517, 367

Bradshaw, C. F., Titarchuk, L., \& Kuznetsov, S. 2007, ApJ, 663, 1225

Branduardi-Raymont, G., Ercan, E. N., \& Chiappetti, L. 1984, A\&A, 130, 175

Chiappetti, L., Treves, A., Branduardi-Raymont, G., et al. 1990, ApJ, 361, 596

Costantini, E., Freyberg, M. J., \& Predehl, P. 2005, A\&A, 444, 187

D'Amico, F., Heindl, W. A., Rothschild, R. E., \& Gruber, D. E. 2001, ApJ, 547, L147

Dickey, J. M., \& Lockmann, F. J. 1990, ARA\&A, 28, 215

Di Salvo, T., \& Stella, L. 2002, in Proc. of the XXXVIIth Rencontres de Moriond, The Gamma-Ray Universe, ed. A. Goldwurm, D. N. Neumann, \& J. Tran Thanh Van, 67
Di Salvo, T., Stella, L., Robba, N. R., et al. 2000, ApJ, 544, L119 Di Salvo, T., Robba, N. R., Iaria, R., et al. 2001, ApJ, 554, 49

Di Salvo, T., Farinelli, R., Burderi, L., et al. 2002, A\&A, 386, 535 (DS02)

Di Salvo, T., Goldoni, P., Stella, L., et al. 2006, ApJ, 649, L91

Falanga, M., Götz, D., Goldoni, P., et al. 2006, A\&A, 458, 21

Farinelli, R., Frontera, F., Zdziarski, A. A., et al. 2005, A\&A, 434, 25

Farinelli, R., Titarchuk, L., \& Frontera, F. 2007, ApJ, 662, 1167 (F07)

Farinelli, R., Titarchuk, L., Paizis, A., \& Frontera, F. 2008, ApJ, 680, 602 (F08)

Frontera, F., Costa, E., dal Fiume, D., et al. 1997, A\&AS, 122, 357

Frontera, F., dal Fiume, D., Malaguti, G., et al. 1998, in The Active X-ray Sky: Results from BeppoSAX and RXTE, ed. L. Scarsi, H. Bradt, P. Giommi, \& F. Fiore, 286

Hasinger, G., van der Klis, M., Ebisawa, K., Dotani, T., \& Mitsuda 1990, A\&A, 235,131

Inogamov, N. A., \& Sunyaev, R. A. 1999, AstL, 25, 269

Kuulkers, E., Parmar, A. N., Owens, A., Oosterbroek, T., \& Lammers, U. 1997, A\&A, 323, 29

Laurent, P., \& Titarchuk, L. 1999, ApJ, 511, 289

Laurent, P., \& Titarchuk, L. 2001, ApJ, 562, L67

Laurent, P., \& Titarchuk, L. 2007, ApJ, 656, 1056

Lavagetto, G., Iaria, R., di Salvo, T., et al. 2004, Nucl. Phys. B, Proc. Suppl., 132,616

Ling, J. C., Wheaton, W. A., Mahoney, W. A., et al. 1996, A\&AS, 120, 677

Maurer, G. S., Johnson, W. N., Kurfess, J. D., \& Strickman, M. S. 1982, ApJ, 254,271

Mitsuda, K., Inoue, H., Koyama, K., et al. 1984, PASJ, 36, 741

Mitsuda, K., Inoue, H., Nakamura, N., \& Tanaka, Y. 1989, PASJ, 41, 97

Paizis, A., Ebisawa, K., Tikkanen, T., et al. 2005, A\&A, 443, 599

Paizis, A., Farinelli, R., Titarchuk, L., et al. 2006, A\&A, 459, 187 (P06)

Penninx, W., Lewin, W. H. G., Zijlstra, A. A., Mitsuda, K., \& van Paradijs, J. 1988, Nature, 336, 146

Peterson, L. E. 1973, in X- and Gamma-Ray Astronomy, ed. H. Bradt, \& R. Giacconi, IAU Symp., 55

Shaposhnikov, N., \& Titarchuk, L. 2007, ApJ, 663, 445

Smale, A. P., Done, C., Mushotzky, R. F., et al. 1993, ApJ, 410, 796

Smale, A. P., Angelini, L., White, N. E., Mitsuda, K., \& Dotani, T. 1994, BAAS, 26,1484

Shrader, C., \& Titarchuk, L. 1998, ApJ, 499, L31

Takei, Y., Fujimoto, R., Mitsuda, K., \& Onaka, T. 2002, ApJ, 581, 307

Titarchuk, L., \& Lyubarskij, Y. 1995, ApJ, 450, 876

Titarchuk, L., \& Shaposhnikov, N. 2005, ApJ, 626, 298

Titarchuk, L., Mastichiadis, A., \& Kylafis, N. D. 1996, A\&AS, 120, 171

Titarchuk, L., Mastichiadis, A., \& Kylafis, N. D. 1997, ApJ, 487, 834

Titarchuk, L., Kuznetsov, S., \& Shaposhnikov, N. 2007a, ApJ, 667, 404

Titarchuk, L., Shaposhnikov, N., \& Arefiev, V. 2007b, ApJ, 660, 556

van der Klis, M. 2001, ApJ, 561, 943

Vrtilek, S. D., Swank, J. H., \& Kallman, T. R. 1988, ApJ, 326, 186

Vrtilek, S. D., Raymond, J. C., Garcia, M. R., et al. 1990, A\&A, 235, 162

White, N. E., Peacock, A., Hasinger, G., et al. 1986, MNRAS, 218, 129

White, N. E., Stella, L., \& Parmar, A. 1988, ApJ, 324, 363

Winkler, C., Courvoisier, T. J.-L., Di Cocco, G., et al. 2003, A\&A, 411, L1 